University of South Carolina

Scholar Commons

7-15-2002

\title{
Electronic Transport Through a Nuclear-Spin-Polarization-Induced Quantum Wire
}

Yuriy V. Pershin Dr

University of South Carolina - Columbia, pershin@physics.sc.edu

S. N. Shevchenko

I. D. Vagner

P. Wyder

Follow this and additional works at: https://scholarcommons.sc.edu/phys_facpub

Part of the Physics Commons

\section{Publication Info}

Published in Physical Review B, ed. Gene D. Sprouse, Volume 66, Issue 3, 2002, pages 035303-1-035303-5.

Pershin, Y. V., Shevchenko, S. N., Vagner, I. D., \& Wyder, P. (2002). Electronic transport through a nuclearspin-polarization-induced quantum wire. Physical Review B, 66(3), 035303-1 - 035303-5. DOI: 10.1103/ PhysRevB.66.035303

(c) Physical Review B, 2002, American Physical Society

This Article is brought to you by the Physics and Astronomy, Department of at Scholar Commons. It has been accepted for inclusion in Faculty Publications by an authorized administrator of Scholar Commons. For more information, please contact digres@mailbox.sc.edu. 


\title{
Electronic transport through a nuclear-spin-polarization-induced quantum wire
}

\author{
Yu. V. Pershin, ${ }^{1,2}$ S. N. Shevchenko, ${ }^{1,3}$ I. D. Vagner, ${ }^{1,2,4}$ and P. Wyder ${ }^{1}$ \\ ${ }^{1}$ Grenoble High Magnetic Fields Laboratory, Max-Planck-Institute für Festkorperforschung and CNRS, BP 166, \\ F-38042 Grenoble Cedex 9, France \\ ${ }^{2}$ Department of Physics, Clarkson University, Potsdam, New York 13699-5820 \\ ${ }^{3}$ B. I. Verkin Institute for Low Temperature Physics and Engineering, 47 Lenin Avenue, 61103 Kharkov, Ukraine \\ ${ }^{4}$ Holon Academic Institute of Technology, 52 Golomb Street, Holon 58102, Israel
}

(Received 12 December 2001; published 1 July 2002)

\begin{abstract}
Electron transport in a low-dimensional structure - the nuclear-spin-polarization-induced quantum wire is theoretically studied. In the proposed system the local nuclear-spin polarization creates the effective hyperfine field that confines the electrons with the spins opposite to the hyperfine field to the regions of maximal nuclear-spin polarization. The influence of the nuclear-spin relaxation and diffusion on the electron energy spectrum and on the conductance of the quantum wire is calculated and the experimental feasibility is discussed.
\end{abstract}

DOI: 10.1103/PhysRevB.66.035303

PACS number(s): 72.25.-b, 73.23.-b, 75.40.Gb

There has been much recent theoretical ${ }^{1-3}$ and experimental $^{4-7}$ interest in the peculiarities of the electron transport in mesoscopic systems with highly polarized nuclear spins. In Ref. 1 a new class of phenomena: the mesonucleo-spinics was proposed based on the strong influence of the hyperfine magnetic field of the polarized nuclei on the energy spectrum and the wave functions of the conduction electrons in few channel mesoscopic systems. It was shown there and later discussed in Ref. 2 that the inhomogeneous external hyperfine magnetic field, acting on the charge carriers confined to move in a ring, influence the quantum interference (mesoscopic) phenomena and can induce the persistent current with some interesting physical features. In Ref. 3 the electron states in a quantum dot with a nuclear-spin polarization were studied within the perturbation theory. Moreover, it was proposed to use the inhomogeneous magnetic field to create so-called magnetic structures, such as magnetic quantum dots, rings, superlattices etc. (see for a review Ref. 8).

The conductance quantization, in the case of the ballistic transport through quantum wires at low temperatures, is by now well studied both experimentally and theoretically. ${ }^{9-11}$ The dependence of the conductance at "zero" temperature on the number of transverse modes in the conductor is given by the Landauer formula: ${ }^{12}$

$$
G=\frac{2 e^{2}}{h} M T,
$$

where $T$ is the average electron transmission probability, $M$ is the number of the transverse modes and the factor " 2 " stands because of the spin degeneracy. It is assumed that the transition probability $T$ is independent of the energy in a small interval between the chemical potentials of the reservoirs. Usually, the number of the transverse modes, defined by the effective width of the conductor, is controlled by the gate voltage and the conductance is changed in discrete steps $2 e^{2} / h .^{11}$

In this paper we propose a different class of experiments, based on the creation of a quantum wire by the hyperfine magnetic field of polarized nuclei acting on the conductionelectron spins. The time evolution of the hyperfine magnetic field, due to the nuclear-spin relaxation and the nuclear-spin diffusion, leads to variation of the number of transverse modes and corresponding electron energies at a constant gate potential that can be directly measured by transport experiments.

The proposed system is depicted in Fig. 1. The twodimensional electron gas (2DEG) is splitted in two parts by a potential applied to the gate electrode (of the width $L$ ) located under 2DEG. Two parts of 2DEG are connected by a nuclear-spin-polarization-induced quantum wire (NSPIQW) created either by the optical nuclear-spin polarization. ${ }^{13-15}$ or by the transport polarization ${ }^{5,6}$ or by any other experimentally suitable method. The chemical potentials of these 2DEG regions are $\mu_{1}$ and $\mu_{2},\left(\mu_{1}-\mu_{2}\right) /|e|$ is the bias voltage. We assume that the bias voltage is small in comparison with the potential that confines the electrons. The conduc-

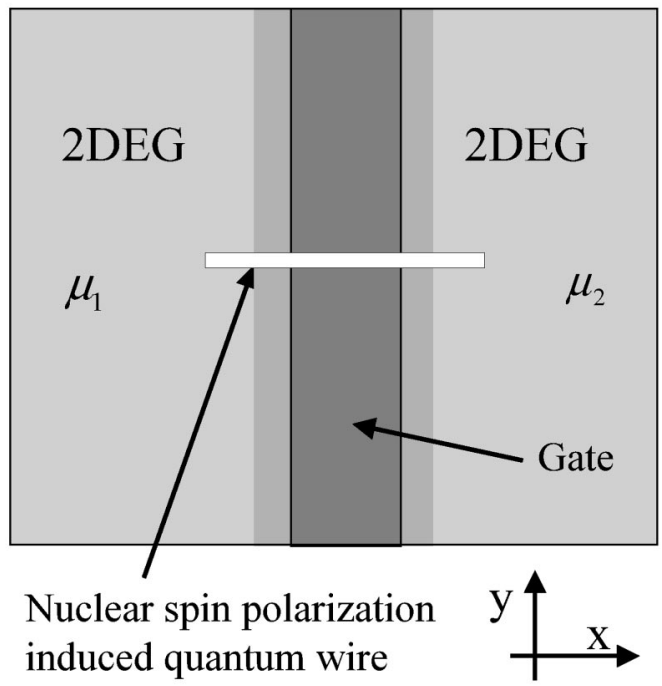

FIG. 1. The geometry of the proposed experiment: the 2DEG is splitted by the gate potential, the narrow conductor is created by the local nuclear-spin polarization. 
tance of the NSPIQW depends on the number of the transverse modes below $\mu_{1}$ and $\mu_{2}$. Since the number of the transverse modes changes in time, the current between two regions of $2 \mathrm{DEG}$ is also time dependent.

The contact hyperfine interaction between an electron and nuclear spins is described by the Fermi contact Hamiltonian ${ }^{16} H_{h f}(\mathbf{r})=(8 \pi / 3) \mu_{\mathrm{B}} \gamma_{n} \hbar \sum_{i} \mathbf{I}_{i} \boldsymbol{\sigma} \delta\left(\mathbf{r}-\mathbf{r}_{i}\right)$, where $\mu_{\mathrm{B}}$ is the Bohr magneton, $\mathbf{I}_{i}$ and $\boldsymbol{\sigma}$ are nuclear and electron spins, and $\mathbf{r}_{i}$ and $\mathbf{r}$ are the radius vectors of the $i$ th nucleus and of the electron. It follows that once the nuclear spins are polarized, the charge-carrier spins feel the effective hyperfine field $\mathrm{B}_{h f}$, which lifts the spin degeneracy. In GaAs/AlGaAs heterojunction one may achieve the hyperfine field of several Teslas. ${ }^{6,4}$ The spin splitting $\left(\mu_{\mathrm{B}} B_{h f}\right)$ due to such hyperfine magnetic field is comparable to the Fermi energy of 2DEG. Thus, if the gate potential $U_{\text {gate }}$ is more than $\mu_{1,2}$, then all the electrons in the region, where nuclear-spins are polarized, will occupy the energetically more favorable states with the spins opposite to $\mathrm{B}_{h f}$. And, furthermore, the nuclear polarization acts on the electrons as the effective confining potential $V_{\text {conf }}=-\mu_{\mathrm{B}} B_{h f}$. This effective confining potential can be used to create different nanostructures with polarized electrons in them. Here we restrict our consideration to NSPI QW.

There are two main mechanisms leading to the time dependence of the hyperfine, field: the nuclear-spin relaxation and the nuclear-spin diffusion. We assume that the nuclearspin polarization is homogeneous in $x$ and $z$ directions. Then the hyperfine field evolution is described by the onedimensional diffusion equation with taking into account the relaxation processes:

$$
\frac{\partial B_{h f}}{\partial t}=D \frac{\partial^{2} B_{h f}}{\partial y^{2}}-\frac{1}{T_{1}} B_{h f},
$$

where $D$ is the spin-diffusion coefficient and $T_{1}$ is the nuclear-spin-relaxation time. ${ }^{17}$ Here we assume that the nuclear-spin polarization is inhomogeneous across the NSPIQW and quite homogeneous along it (at least in depleted region), provided that the length of nuclear-spinpolarized region is larger then the depleted region. In this case the diffusion in $x$ direction from ends is irrelevant for the properties of NSPIQW.

Let us discuss experimental feasibility of this assumption. Using the method of optical nuclear spin-polarization, ${ }^{13-15}$ the sample is illuminated locally by, for example, putting a mask on it. The resolution of the optical illumination of the sample can be high enough. Usual optic technique allows one to create the light beams of the width of the order of the wave length $(\sim 500 \mathrm{~nm})$, by using near fields optics the beamwidth can be sufficiently reduced $(\sim 100 \mathrm{~nm})$. Hence a NSPIQW of the width of $1 \mu \mathrm{m}$ can be created by the modern experimental technique. In semiconductor heterostructures having supreme quality, the electron mean free path can be as large as $100 \mu \mathrm{m}$ and NSPIQW will operate in the quantum regime. We assume the initial condition to be of the Gaussian form: $B_{h f}(y, 0)=B_{0} \exp \left(-y^{2} / 2 d^{2}\right)$. The two parameters, $d$ and $B_{0}$, define the half-width and the amplitude of the initial distribution of the hyperfine field, respectively. Then the solution of Eq. (2) is:

$$
B_{h f}(y, t)=B_{0} e^{-t / T_{1}}\left(1+\frac{t}{t_{0}}\right)^{-1 / 2} e^{-y^{2} /\left[2 d^{2}\left(1+\left(t / t_{0}\right)\right)\right]},
$$

where $t_{0}=d^{2} / 2 D$.

The microscopic description is based on the following Hamiltonian:

$$
H=-\frac{\hbar^{2}}{2 m^{*}} \Delta+V(x)+U(z)+\mu_{\mathrm{B}} \boldsymbol{\sigma} \mathbf{B}_{h f}(y, t) .
$$

Here $m^{*}$ is the electron effective mass and $V(x)$ is the potential energy associated with the gate. The form of the potential $V(x)$ is not very important in our consideration. It defines the transmission probability in Eq. (1). If we take $V(x)=V_{0}=$ const for $|x|<L / 2$ and $V(x)=0$ otherwise, then $T=1$. We suppose, as it is usually done for $2 \mathrm{DEG}$, that only the lowest subband, corresponding to the confinement in $z$ direction, is occupied and we can ignore the higher subbands. Thus, we omit in the following $z$ dependence of the wave function. The nuclear-spin-relaxation time $T_{1}$ and the characteristic diffusion time $t_{0}$ in semiconductors at sufficiently low temperatures is rather long. They vary from several hours to few minutes. ${ }^{14}$ Thus the time scale introduced by nuclear-spin system is several orders of magnitude larger than the time scale of typical electron equilibration processes. In such a case the conduction electrons see a quasiconstant nuclear field. This simplifies the calculation by avoiding the complications that would appear when solving the Schrödinger equation with the time dependence due to polarized nuclei. Taking into account the electrons only with opposite to the hyperfine field spins (for which the effective potential is attractive), we obtain the following equation for the transverse-modes energy spectrum:

$$
-\frac{\hbar^{2}}{2 m^{*}} \frac{\partial^{2} \psi(y)}{\partial y^{2}}-\mu_{\mathrm{B}} B_{h f}(y, t) \psi(y)=\varepsilon^{t r} \psi(y) .
$$

To proceed we approximate the hyperfine field (3) by

$$
\widetilde{B}_{h f}=\frac{1}{\mu_{\mathrm{B}}} \frac{U_{0}}{\cosh ^{2}(\alpha y)},
$$

connected with Eq. (3) by the relations:

$$
\begin{gathered}
B_{h f}(0, t)=\widetilde{B}_{h f}(0, t), \\
\int B_{h f}(y, t) d y=\int \widetilde{B}_{h f}(y, t) d y .
\end{gathered}
$$

It follows from Eq. (7) that both fields at $y=0$ have the same value, as well as the areas under the curves $B_{h f}(y, t)$ and $\widetilde{B}_{h f}(y, t)$ at any fixed $t$. This provides the total nuclear-spin polarization to be the same for the two fields. From Eqs. (7) we obtain $U_{0}=\mu_{\mathrm{B}} B_{0} e^{-t / T_{1}}\left[1+\left(t / t_{0}\right)\right]^{-1 / 2}$ and $\alpha^{-1}$ $=d \sqrt{\pi / 2}\left[1+\left(t / t_{0}\right)\right]^{1 / 2}$. From Fig. 2 it is clearly seen that the hyperfine fields given by Eqs. (3) and (6) have very similar dependence on $y$. Substituting the hyperfine field, Eq. (6), 


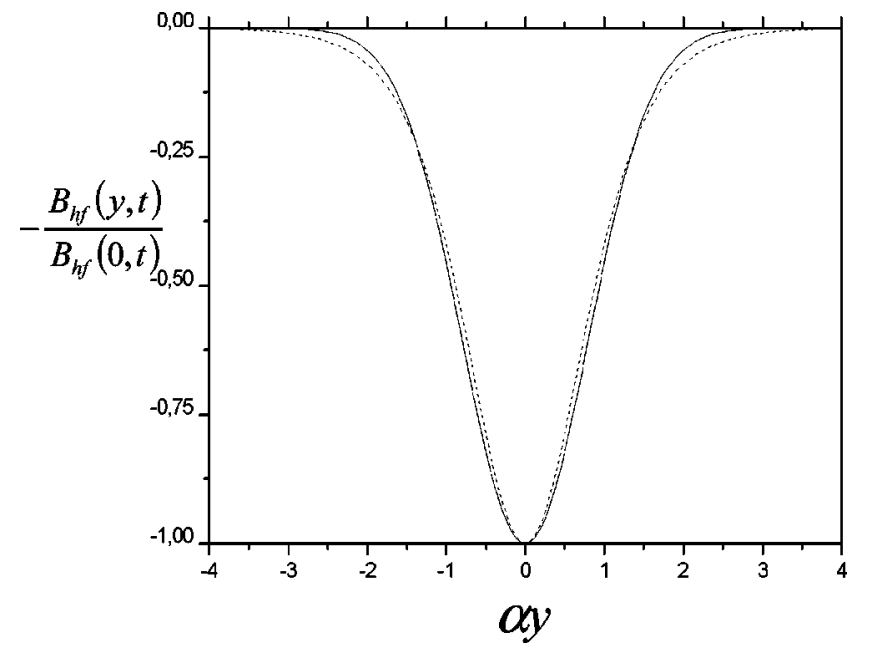

FIG. 2. Comparison of the exact hyperfine field (solid line) and the model hyperfine field (dashed line).

into Eq. (5), we obtain the one-dimensional Schrödinger equation with the modified Pöschl-Teller potential:

$$
-\frac{\hbar^{2}}{2 m^{*}} \frac{\partial^{2} \psi(y)}{\partial y^{2}}-\frac{U_{0}}{\cosh ^{2}(\alpha y)} \psi(y)=\varepsilon^{t r} \psi(y) .
$$

Solution of Eq. (8) can be expressed in terms of the hypergeometric function ${ }^{18}$ and the energy spectrum is

$$
\varepsilon_{n}^{t r}=-\frac{\hbar^{2} \alpha^{2}}{2 m^{*}}(s-n)^{2},
$$

where $\left.s=\frac{1}{2}\left[-1+\sqrt{1+\left(8 m^{*} U_{0} / \hbar^{2} \alpha^{2}\right.}\right)\right]$ and $n=0,1,2, \ldots$. The number of the energy levels $N$ is finite and, being defined by the condition $n<s$, is given by $N=1+[s]$, where $[\cdots]$ denotes the integer part. A level appears or disappears in the system when $s(t)$ becomes an integer.

Let us consider the time dependence of the parameter $s$ in more detail. The expression for $s$ can be rewritten in a more convenient form:

$$
s(t)=\frac{1}{2}\left[-1+\left(1+C e^{-t / T_{1}} \sqrt{1+\frac{t}{t_{0}}}\right)^{1 / 2}\right],
$$

where the dimensionless parameter $C=4 \pi\left(m^{*} d^{2} / \hbar^{2}\right) \mu_{\mathrm{B}} B_{0}$ contains only the initial distribution parameters $d$ and $B_{0}$. There are two different characteristic times in Eq. (10): the diffusion characteristic time $t_{0}$ and the relaxation characteristic time $T_{1}$. It is easy to see that $s$ has a maximum at $t_{\max }$ $=\left(T_{1} / 2\right)-t_{0}$. We can distinguish three regimes: the diffusive regime $\left(T_{1} \gg t_{0} \sim t\right)$, the relaxation regime $\left(T_{1}<2 t_{0}\right)$, and the intermediate regime $\left(T_{1} \gtrsim 2 t_{0}\right)$. Here $t$ is the observation time. Time dependences of the number of the energy levels $N$ in different regimes are shown in Fig. 3. In the diffusive regime (full line) the number of levels increases with time, while in the relaxation regime (dashed line) the number of levels decreases with time. In the intermediate regime (dotted line) there is the maximum of the function $s(t)$ at $t$ $=t_{\max }$. The estimation of the number of the transverse energy levels for the following set of parameters: $B_{0}=1 \mathrm{~T}$,

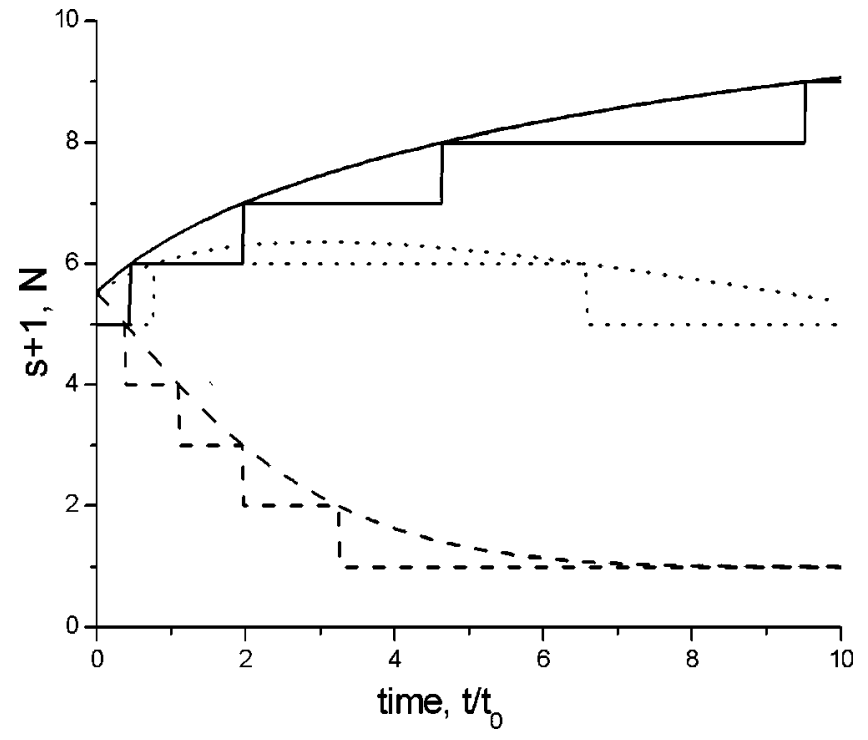

FIG. 3. Different kinds of the level number dependences on time $N=N(t)$ and $s=s(t)$ for $C=100$ : solid line-diffusive regime $\left(T_{1} / t_{0}=80\right)$; dashed line-relaxation regime $\left(T_{1} / t_{0}=1\right)$; dotted line-intermediate regime $\left(T_{1} / t_{0}=8\right)$. The stepped lines correspond to the level number $N$, the smooth lines to $s+1$.

$d=1 \mu \mathrm{m}, m^{*}=0.067 m_{e}$ gives us $N(t=0)=13$. We can also estimate the number of electrons per unit length: $n_{L}$ $=(1 / \pi \hbar) \Sigma \sqrt{2 m^{*}\left|\varepsilon_{n}^{t r}\right|}=(\alpha / \pi) N\left[s-\frac{1}{2}(N-1)\right]$, which at $t$ $=0$ and $N \gg 1$ gives $n_{L} \simeq\left(1 / \sqrt{2} \pi^{3 / 2}\right)\left(N^{2} / d\right)$. For $N=13$ and $d=1 \mu \mathrm{m}$ we have $n_{L} \simeq 2 \times 10^{5} \mathrm{~cm}^{-1}$.

The time dependence of the transverse energy levels in the diffusive regime is shown in Fig. 4(a). The chemical potentials $\mu_{1}$ and $\mu_{2}$ are depicted by the horizontal lines. The number of transverse energy levels below them at the moment of time $t$ is the number $M$ of the transverse modes. The points of intersection of the chemical potentials with the transverse energy levels are given by

$$
\mu_{1,2}=V_{0}-\varepsilon_{n}^{t r}(t)
$$

An interesting feature of the transverse energy spectrum is that the energy of some levels [for example, the level with $n=3$ in Fig. 4(a)] at the short times decreases until it has a minimum. In the relaxation regime [Fig. 4(b)] the absolute values of the energy levels and the number of the levels decrease monotonically in time. At large times only the energy level with $n=0$ survives. In the intermediate regime a mixing of the relaxation and diffusion regimes happens [Fig. $4(c)]$. At short times the energy-level behavior is determined by the nuclear-spin diffusion and at large times by the nuclear-spin relaxation.

The dependence of the conductance on the time is shown in Figs. 4(d)-4(f). When an energy level crosses the chemical potentials [the time of the intersection is given by Eq. (11)] the conductance changes by $e^{2} / h$. We underline that due to the spin selective effective potential the height of the conductance steps is just $e^{2} / h$, which is a half of the conductance quantum $G_{0}=2 e^{2} / h$. 
a)

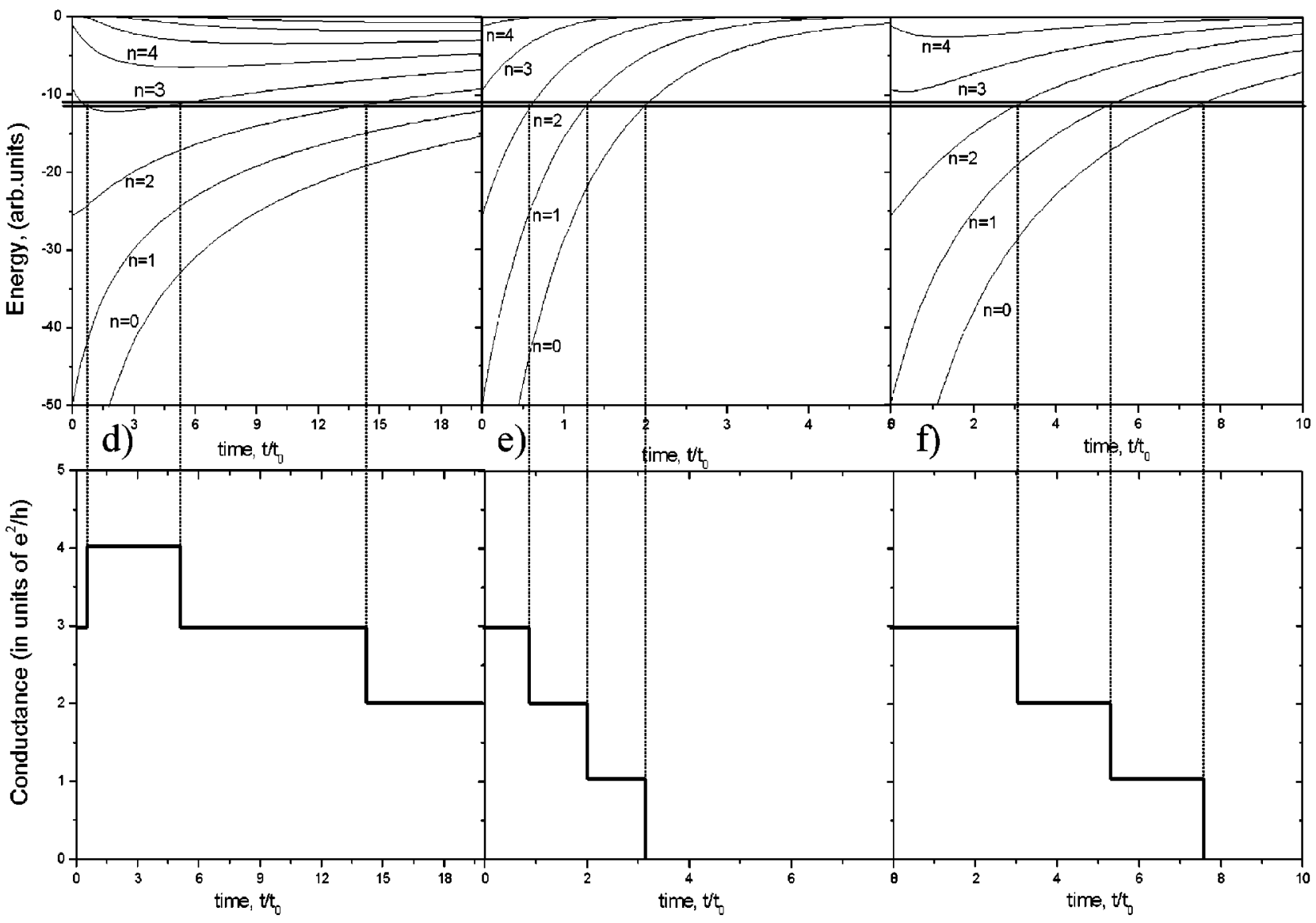

FIG. 4. Time evolution of the energy levels in the NSPIQW: (a) The diffusive regime; (b) relaxation regime; and (c) intermediate regime, and $(\mathrm{d}-\mathrm{f})$ respective time dependences of the conductance. The parameters of calculations are the same as in Fig. 3.

It is quite clear that sharp conductance quantization steps shown in Figs. 4(d)-4(f) will be smoothed in real experiment. The main mechanisms of smoothing are: the effect of finite temperature; scattering of electrons on impurities and defects; and inhomogeneity of the initial hyperfine field profile along the wire. The temperature smoothing can be eliminated if the experiment is performed at low enough temperature, when the distance between the transverse mode subbands is much larger then $k_{B} T$, which for $1 T$ hyperfine field is of order of $10-100 \mathrm{mK}$. The using of high-quality samples will reduce the influence of scattering. The conditions of homogeneity of the wire were discussed before.

Let us consider the lifetime of the NSPIQW. It can be defined by the following condition: $\left|\varepsilon_{0}^{t r}\left(t_{l}\right)\right|=k_{\mathrm{B}} T^{*}$, where $k_{\mathrm{B}}$ is the Boltzmann's constant and $T^{*}$ is the temperature. Using Eq. (9), we calculate the time $t_{l}$ for two limiting cases: $T_{1} \ll t_{0}$ and $T_{1} \gg t_{0}$. In the first case (the strong relaxation limit) $t_{l} \sim T_{1} / 2 \ln \left[m^{*} d^{2}\left(\mu_{\mathrm{B}} B_{0}\right)^{2} / \hbar^{2} k_{\mathrm{B}} T^{*}\right]$. In the second case (the diffusion regime) $t_{l} \sim t_{0}\left(\mu_{\mathrm{B}} B_{0} / k_{\mathrm{B}} T^{*}\right)^{2}$. Let us estimate the half-width of the wire $\alpha^{-1}$ at $t \sim t_{l}$. For $T^{*}=30 \mathrm{mK}$ and
$B_{0}=1 \quad \mathrm{~T}$ we have $\alpha^{-1} \sim d$ for $T_{1} \ll t_{0}$ and $\alpha^{-1} \sim 20 d$ for $T_{1} \gg t_{0}$.

To summarize, a different system for investigation of 1D electron transport-the nuclear-spin-polarization-induced quantum wire-is proposed. We investigate the influence of the nuclear-spin relaxation and diffusion on the properties of the electron system. The time dependences of the electron energy spectrum and of the conductance of the quantum wire are studied. We expect that the experimental study of the described system can give some information, such as the nuclear-spin-diffusion coefficient $D$ and the nuclear-spin relaxation time $T_{1}$. Furthermore, we note that the method of local nuclear-spin polarization allows us to create different low-dimensional nuclear-spin-polarization-induced quantum structures (quantum dots, rings, wires, etc.) using the same sample and different illumination masks.

We acknowledge useful discussions with V. Fleurov, V. Ivanov, F. Peeters, and V. Privman. This research was supported in part by the US National Science Foundation, Grant No. DMR-0121146 and ECS-0102500, and in part by EU, Grant No. IST-2000-29686. 
${ }^{1}$ I.D. Vagner, A.S. Rozhavsky, P. Wyder, and A.Yu. Zyuzin, Phys. Rev. Lett. 80, 2417 (1998).

${ }^{2}$ V.A. Cherkaskiy, S.N. Shevchenko, A.S. Rozhavsky, and I.D. Vagner, Low Temp. Phys. 25, 541 (1999).

${ }^{3}$ V. Fleurov, V. A. Ivanov, F.M. Peeters, and I.D. Vagner, Physica E (to be published), cond-mat/0109271.

${ }^{4}$ A. Berg, M. Dobers, R.R. Gerhardts, and K.v. Klitzing, Phys. Rev. Lett. 64, 2563 (1990).

${ }^{5}$ B.E. Kane, L.N. Pfeiffer, and K.W. West, Phys. Rev. B 46, 7264 (1992).

${ }^{6}$ K.R. Wald, L.P. Kouwenhoven, P.L. McEuen, N.C. van der Vaart, and C.T. Foxon, Phys. Rev. Lett. 73, 1011 (1994).

${ }^{7}$ S. Kronmuller, W. Dietsche, K.v. Klitzing, G. Denninger, W. Wegscheider, and M. Bichler, Phys. Rev. Lett. 82, 4070 (1999).

${ }^{8}$ F.M. Peeters and J. De Boeck, in Handbook of Nanostructured Materials and Nanotechnology, edited by H.S. Nalwa (Academic Press, New York, 1999), Vol. 3, p. 345.

${ }^{9}$ B.J. van Wees, H. van Houten, C.W.J. Beenakker, J.R. Williamson, L.P. Kouwenhoven, D. van der Marel, and C.T. Foxon, Phys. Rev. Lett. 60, 848 (1988).

${ }^{10}$ D. Wharam, T.J. Thornton, R. Newbury, M. Pepper, H. Ahmed,
J.E.F. Frost, D.G. Hasko, D.C. Peacock, D.A. Ritchie, and G.A.C. Jones, J. Phys. C 21, L209 (1988).

${ }^{11}$ S. Datta, Electronic Transport in Mesoscopic Systems (Cambridge University Press, Cambridge, England, 1997).

${ }^{12} \mathrm{R}$. Landauer, in Localization, Interaction and Transport Phenomena, edited by G. Bergmann and Y. Bruynseraede (Springer, New York, 1985).

${ }^{13}$ G. Lampel, Phys. Rev. Lett. 20, 491 (1968).

${ }^{14}$ Optical Orientation, edited by F. Meier and B. P. Zakharchenya (Elsevier, Amsterdam, 1984).

${ }^{15}$ S.E. Barrett, R. Tycko, L.N. Pfeiffer, and K.W. West, Phys. Rev. Lett. 72, 1368 (1994); For a review see R. Tycko, S.E. Barrett, G. Dabbagh, L.N. Pfeiffer, and K.W. West, Science 268, 1460 (1995).

${ }^{16}$ C.P. Slichter, Principles of Magnetic Resonance, 2nd ed. (Springer-Verlag, Berlin, 1991).

${ }^{17}$ D. Wolf, Spin-Temperature and Nuclear-Spin Relaxation in Matter (Clarendon Press, Oxford, 1979).

${ }^{18}$ L.D. Landau and E.M. Lifshitz, Quantum Mechanics (NonRelativistic Theory), 3rd ed. (Pergamon, London, 1977). 